\title{
Evoluation of the effect of structural-physical changes in water on biological activity
}

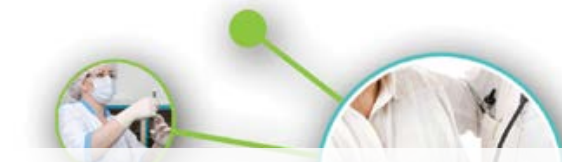

The paper examines the influence of changes in structural and physical state of water on biological activity of test organisms (luminescent bacteria of the transgenic strain Escherichia coli K12 TG1).). The preliminary informational effect of UHF radiation on water shifts the energy distribution in water associates, which causes a growth spurt of microorganism activity by more than a decimal exponent. The paper also shows that the energy source for ATP synthesis, produced by proton ATP synthase (FOF1-ATP synthase, where F1 and F0 are protein complexes), is connected with a nonlocal electron transfer from water to the hydrate structures of the F0-complex, which, by way of rotation, transfers protons for ATP synthesis.

Keywords: associate water phase, UHF radiation, electromagnetic emission, E. Coli test system, peroxide anion radical. Abbreviations: ER - electromagnetic radiation, UHF - ultra-high frequency, HF - high frequency, LFE - low
frequency emission, NIR - non-ionizing radiation

\section{Introduction}

Non-ionizing electromagnetic radiation in context of its biological effectsis a closely studied phenomenon [1-3]. The wide span of non-ionizing radiation (NIR) ranges that affect biosystems, from subhertz to terahertz frequencies, the intensity, the correlation of biological effects with changes in organisms water state, and long-term manifestations, call for studyingthe affect between altered metastable states and biological activity of water subjected to NIR pretreatment.

The previous studies [4] have shown that water, as a superconducting system with selforganizing critical behavior, is hyper sensitive to weak variable signals. Additionally, external electrical and/or electromagnetic perturbations of the water environment informationally impact

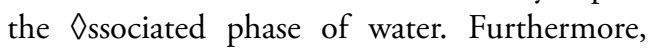
the electrophysical state of the phase possesses properties of self-renewal and self-reproduction of its state as a result of metabolic processes between itself and the environment.

Thus, an indirect (after the information processing of water by UHF radiation) impact on a living organism of water with altered structural and physical organization of the associated water phase allows to establish the link between observed biological responses and structural and physical changes in water, as well as with the low-intensity electromagnetic radiation.

\section{Materials and Methods}

Parametrization of water state as an electromagnetically and chemically active system can be carried out by utilizing changes in its volumetric properties (for example, by means of infrared absorption spectroscopy) and the state of associated water phases present in form of ion-crystal associates [5]. The associated water phase, an amorphous ice VI and VII, stabilized by delocalized charges and nanosized voids, is significantly different in dispersion and thermodynamic properties.

Microphysical parameters of associated water can be obtained from the proton magnetic resonance data [6], spontaneous Raman scattering spectra [7], fluorescence of electronexcited oxygen and its dimers $[8,9]$, according to microwave absorption and emission (orthoand para- molecules of water and hydroxyl have rotational resonances at frequencies of 0.072 $\mathrm{cm}^{-1}(2.16 \mathrm{GHz})$ and $0.057 \mathrm{~cm}^{-1}(1.72 \mathrm{GHz})$ [10].

\author{
AA Stekhin ${ }^{1}$, \\ GV Yakovleva ${ }^{1}$, \\ TI Iksanova ${ }^{1}$, \\ KN Pronko², \\ VM Zemskov*2

\section{${ }^{1}$ Center for Strategic Planning and Management of Medical and} \\ Biological Health RisksMoscow, Russia
}

\section{${ }^{2}$ Department of Research and Development at Facecontrol, Inc., Florida, Miami, USA}

*Author for correspondence:

vzemskov@facecontrol.biz 
Despite the advantages, the use of spectral methods for parametrization of the associated water phase, including a promising lowfrequency dielectric measurement method [11], is rather limited due to the phase being prone to uncontrollable changes when exposed to radiation.

Similar instrument-methodical limitations also arise when using liquid chromatography to separate water associates. The method allows to separately observe molecular clusters of hydrogen peroxide in a monomeric, dimeric and trimeric form (refractive index $\mathrm{n}>1.34$ ) and associates $\left(\left[\mathrm{OH}^{-}\left(^{*}\right)\left(\mathrm{H}_{2} \mathrm{O}_{\mathrm{p}}^{\int}\right]_{\mathrm{q}},\left[\mathrm{OH}^{-(*)} \ldots \subseteq \in_{2}^{-(*)}\left(\subseteq_{2} \in\right)_{\mathrm{p} 2}^{\jmath}\right]_{\mathrm{q}^{2}}\right.\right.$ in water using a refractometric detector, where $t$ represents the ice tetragonal structure (Wolrafen pentameter), $\mathrm{n}$ is a parameter of molecular filling of the associate with an insertion compound, $p$, $\mathrm{p}^{\prime}\left(\mathrm{q}, \mathrm{q}^{\prime}\right)$ are parameters of ionic coordination (degree of association) before (without an apostrophe) and after (with an apostrophe) the structural organization of the associate is restructured [12]) over a series of peaks with $n$ $<1,34$ (dielectric permittivity of the associated water phase is 36 [13]). However, the prolonged passage of water through a capillary column causes decay or transformation of associates, which makes it impossible to use the method for quantitative measurements of the water associates parameters.

Of all non-perturbing methods of water systems study, kinetic chemiluminescence [12] is a promising one. It is used to study water activity according to the energy state of its peroxide associates (via the concentration and binding energy of radical anions in complexes). This method has not only the highest sensitivity to determine free radicals in water, formed during the decay of anion radicals, but makes it possible to evaluate microstructural changes in the properties of water.

Determination of the mass content in the associated phase $(\mathrm{q}, \%)$ of water was carried out by cryophysical capillary method [5]. The method is based on the ability of associated water to form nanosized voids that are filled with atmospheric gases. The fraction of the void space is $\mathrm{Kh}=0.44 \ldots 0.21 \mathrm{OH}^{-(*)}$ is the ice-VI associate and $\mathrm{K}^{\mathrm{h}}+=0.89 \ldots 0.61-\mathrm{H}+\left(^{*}\right)$ is the ice-VII associate, thus comprising a significant share of the total volume of associates. The obtained values of the volume fraction of voids in associated water allow to determine the mass parameters of the associates, since the subnanosized voids of the associated liquid do not exist in ice crystals. It is the reason why the dissociation of associates releases the air dissolved in them, which forms microvoids in ice crystals as it is released (FIGURE 1).

Cryophysical capillary method allows for drinking waterparametrization by mean and variation values of its mass fraction with an accuracy of $5 \%$ by average value and $12 \%$ by the variational index.

The energy distribution of the associated water phase (the states fraction $\otimes(\mathrm{qi})$, rel. units) is a structural analogue of the size distribution of associates - an indicator determined by the results of individual measurement of the phase fraction in a set of capillaries. The possibility of parameterization of the associated water phase as per the size (energy distribution) is based on the filling of the capillary microchannel with only one chain of self-similar associates, which is a consequence of the quantum behavior of the phase capable of self-reproduction of its structure [12]. The chain structures of the associates in capillaries (the capillary internal diameter is 1.23 $\mathrm{mm}$ ) are more stable than individual associates in optical cells of laser interferometers, where they show phase instability $[12,14]$, and the dimensions of associates increase significantly (up to $100 \mu \mathrm{m}$ ) as compared with the capillary associates $\left(\begin{array}{ll}40 & \mu \mathrm{m}\end{array}\right)$. To informationnaly impact water, a wide-range UHF-radiation, semiconductor injection generator with alloyed layers of indium phosphide ( $\mathrm{InP})$ was used, with a capacity from $10^{-16} \mathrm{~W} / \mathrm{cm}^{2}$ to $10-18 \mathrm{~W} / \mathrm{cm}^{2}$

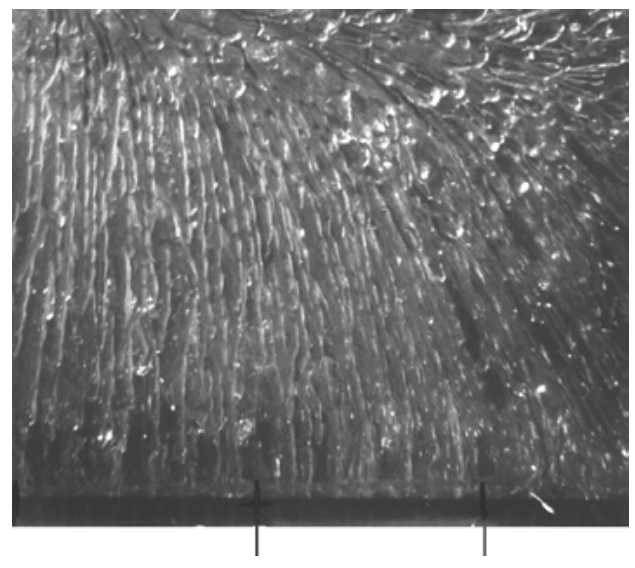

FIGURE 1. The volumetric structure of microvoids distribution in ice crystals formed during the decay of associates upon freezing of physically activated distilled water. 
operating in the frequency range from 40 to 180 $\mathrm{GHz}[15]$.

Radio emission parameters of water exposed to low-intensity UHF-radiation were estimated with EFA-300 electrical tension meter (made in USA).

Biotest systems are an important tool for investigating the nonlinear behavior of associated water phase, which confirms the biological significance of structural and physical changes in water upon its activation. The most studied among them are sensor bioluminescent systems "Ecolum" on the basis of lux operons of luminescent bacteria of Escherichia coli K12 TG1 transgenic strain [3,16-18], which are characterized not only by high sensitivity to environmental factors, but also by high stability of their parameters.

The reason this method was chosen to record changes in the state of water lies in the fact that biological objects have an increased sensitivity to the changes in the electrophysical state of water compared to physical methods of measurements. The biological test facility "Ecolum" (E. Coli microorganisms) is widely used in environmental practice and biological researches, including instances when the cell culture is subject to electromagnetic and acoustic radiation.

When low-intensity UHF-radiation is applied to E. Coli K-12 at frequencies of 70.6 and $73 \mathrm{GHz}$, with a power of $0.06 \mathrm{~mW} /$ $\mathrm{cm}^{2}$, bacterial growth parameters, $\mathrm{pH}$ andthe medium electrical conductivity in the buffer change [2]. Along with this, changes in cell morphology, cell division processes, growth rate and enzyme release into the medium are noted [19]. Organisms may remember biological effects arising from radiation for a long period of time [20].

Changes in the electrochemical state of water were assessed according to the following indicators [12]: Biocatalytic activity (concentration of peroxide anion radicals $\left.\left.\left(\mathrm{HO}_{2}^{-}{ }^{-}\right)\right), \mu \mathrm{g} / \mathrm{L}\right)$ - determined by the chemiluminescence method (liquid analyzer chemiluminescent LIC UNIVERSAL, TU 9443-001-42844321-03), redox potential (h, $\mathrm{mV})$, hydrogen index ( $\mathrm{pH}$, units), electrical conductivity (S, $\mu \mathrm{S} / \mathrm{cm})$.

For research, a weakly mineralized calcium bicarbonate (magnesium, potassium) drinking water of Nestle brand containing excess oxygen $(12.3 \mathrm{mg} / \mathrm{L})$ was used.

\section{Results and Discussion \\ - Dynamics of electrochemical, structural, physical and biological indicators of Nestle water after bottle depressurization}

The initial state of Nestle water after the bottle depressurization was assessed without it being left open. The electrochemical parameters of the water during the study are characterized by significant changes brought about bythe associated water phase transformation processes occurring in water as a result of the diffusion transfer of excess oxygen dissolved in water. Changes in the concentration of dissolved oxygen within the range of $12.3-9.4 \mathrm{mg} / \mathrm{L}$ (TABLE 1) are accompanied by an increase in the hydrogen index (from 7.07 to $7.72 \mathrm{pH}$ units) and redox potential (from 211 to $265 \mathrm{mV}$ ).

The dynamics of changes in the content of peroxide anion radicals in water after the bottle depressurization, presented in FIGURE 2, shows that the production of macro-quantities of oxygen forms in water (by the degree of $1 \mu \mathrm{g} / \mathrm{L}$ ) occurs after 1 hour after contact of water with atmospheric air. However, after 3 hours, their content decreases to a minimum value and is maintained stably for a long time. Such processes in water are probably caused by a restructuring of the associated water phase structure related to the condensation of electrons from the environment. The observed dynamics of active forms of oxygen in water cannot be described by classical equations of chemical kinetics and is rather a consequence of effects of a nonlocal interaction (in time) of a quantum object $[12,14]$.

The structural-physical state of the associated water phase was evaluated after it had been exposed to atmospheric air. Dynamic changes in the state of the associated water phase were registered during a long period of time.

After the water bottle is depressurized, the share of the associated state phase in the sample reaches its maximum within 2 days $\left(\mathrm{q}_{\mathrm{av}}=0,435 \%\right)$ as compared to its value in the initial period and a prolonged exposure afterwards $\quad\left(\mathrm{q}_{\mathrm{av}}=0,389-0,395 \%\right.$ (TABLE 2), which is due to the decay of its high-energy 
Table 1. Changes in electrochemical parameters and concentration of dissolved oxygen in Nestle water after the bottle depressurization.

\begin{tabular}{|c|c|c|c|c|c|c|c|}
\hline \multirow{3}{*}{$\begin{array}{l}\text { Exposure } \\
\text { time* } \\
\text { hours }\end{array}$} & \multicolumn{7}{|c|}{ Parameters } \\
\hline & \multicolumn{2}{|c|}{$\begin{array}{l}\text { Hydrogen index, } \\
\text { pH, units }\end{array}$} & \multicolumn{2}{|c|}{$\begin{array}{c}\text { Redox potential, } \\
\text { Eh, mV }\end{array}$} & \multicolumn{2}{|c|}{$\begin{array}{c}\text { Electrical } \\
\text { conductivity, } \\
S, \mu S / c m\end{array}$} & \multirow{2}{*}{$\begin{array}{c}\mathrm{O}_{2} \text { concentration, } \\
\mathbf{m g} / \mathbf{d m}^{3}\end{array}$} \\
\hline & mean & variations & mean & variations & mean & $\mathrm{T},{ }^{\circ} \mathrm{C}$ & \\
\hline 0 & 7.07 & 0.0006 & 210.9 & 0.0023 & 211.6 & 23.4 & 12.29 \\
\hline 1 & 7.07 & 0.0004 & 218.1 & 0.0039 & 211.2 & 23.2 & 12.10 \\
\hline 2 & 7.03 & 0.0005 & 213.8 & 0.0053 & 211.8 & 23.3 & 10.93 \\
\hline 3 & 7.06 & 0.0006 & 222.4 & 0.0040 & 211.2 & 23.3 & 9.44 \\
\hline 4 & 7.09 & 0.0010 & 221.0 & 0.0025 & 211.6 & 23.3 & 9.43 \\
\hline 5 & 7.14 & 0.0010 & 227.2 & 0.0080 & 212.8 & 23.5 & 9.44 \\
\hline 1 day & 7.42 & 0.0007 & 213.3 & 0.0027 & 212.0 & 23.4 & 9.79 \\
\hline 3 days & 7.72 & 0.0013 & 264.8 & 0.0081 & 210.0 & 22.9 & - \\
\hline \multicolumn{8}{|c|}{ Note ${ }^{*}$ - after the bottle was opened } \\
\hline
\end{tabular}

\begin{tabular}{|c|c|c|c|c|}
\hline $\begin{array}{c}\text { Table 2. Measurements of the share of associated water phase (qav, \%) in nestle water. } \\
\text { Date of } \\
\text { measurement }\end{array}$ & $\begin{array}{c}\text { Water samples } \\
\text { (exposure to air) }\end{array}$ & Temperature, & \multicolumn{2}{|c|}{ Phase share, qav., \% } \\
\hline \multirow{2}{*}{5.10} & Nestle (opened on 5.10) & 23.2 & 0.389 & 0.038 \\
\hline \multirow{2}{*}{17.10} & Nestle (opened on 4.10) & 23.2 & 0.435 & 0.022 \\
\hline \multirow{2}{*}{20.10} & distillate water & 23.1 & 0.695 & 0.037 \\
\cline { 2 - 5 } & Nestle (opened on 29.09) & 23.1 & 0.395 & 0.041 \\
\hline \multirow{2}{*}{25.10} & distillate water & 22.2 & 0.694 & 0.030 \\
\hline & Nestle (opened on 29.09) & 22.2 & 0.396 & 0.035 \\
\hline & Nestle (opened on 23.10) & 22.2 & 0.463 & 0.030 \\
\hline
\end{tabular}

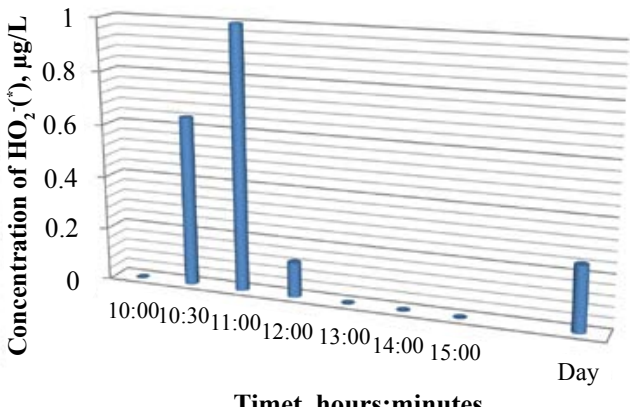

Timet, hours:minutes

FIGURE 2. Dynamics of changes in the concentration of peroxide anion radicals (HO2-(*)) in oxygen-saturated Nestle drinking water after the bottle is depressurized.

states as a result of electrochemical processes.

The analysis of energy distribution of the associated water phase (an analog of the associates distribution as per size) given in TABLE 3 indicates absence of bioenergetically active high-energy states in Nestle water with $\mathrm{q}_{\mathrm{i}}>1,0 \%$. Low presence of peroxide anion radicals in the associated water phase (Nestle water) is seen in their $5 \%$ presence in the range of $\mathrm{q}_{\mathrm{i}}=0,8-1,0 \%$ only on the first and second days after the bottle was opened. With longer exposure of water to the open air, the energy distribution remains stable.

Water samples were held at ambient temperature with no exposure to electromagnetic emitters to determine the effect of background electromagnetic radiation on the EcoLum test culture [21]. It was established on the basis of the studies carried out in [21] that the changes induced by the low-frequency electromagnetic field do not disappear immediately after its termination, but remain for at least 168 hours, which predetermines the need for long-term observations of evolution in the activity.

Our study of the dynamic changes in the "Ecolum" test culture as the water was kept in different conditions (TABLE 4) reveals that both in terms of absolute values of the activity of "Ecolum" microorganisms in a reference distilled water and of relative (to distilled water) values of test culture activity in Nestle water, the changes depend on the storage conditions 
Table 3. Energy distribution (density of states $\Delta$, rel. units) of the associated water phase in Nestle water.

\begin{tabular}{|c|c|c|c|c|c|c|}
\hline \multirow{2}{*}{$\begin{array}{l}\text { Studied } \\
\text { water }\end{array}$} & \multicolumn{6}{|c|}{ Energy levels, ${ }_{\Delta} q_{i} \%$} \\
\hline & $0 \ldots 0,2$ & $0.2 \ldots 0,4$ & $0.4 \ldots 0,6$ & $0.6 \ldots 0,8$ & $0.8 \ldots 1,0$ & $1.0 \ldots 1,2$ \\
\hline \multicolumn{7}{|c|}{$5.10 \mathrm{~T}=23,2^{\circ} \mathrm{C}$ (opened on 5.10) } \\
\hline Nestle & 0.15 & 0.30 & 0.45 & 0.10 & 0 & 0 \\
\hline \multicolumn{7}{|c|}{$5.10 \mathrm{~T}=23,2^{\circ} \mathrm{C}$ (opened on 4.10 ) } \\
\hline Nestle & 0 & 0.30 & 0.60 & 0.05 & 0.05 & 0 \\
\hline \multicolumn{7}{|l|}{ 17.10 $\mathrm{T}=23,1^{\circ} \mathrm{C}$} \\
\hline distillate water & 0.05 & 0.10 & 0.15 & 0.35 & 0.20 & 0.15 \\
\hline Nestle & 0.05 & 0.45 & 0.40 & 0.10 & 0 & 0 \\
\hline \multicolumn{7}{|l|}{$20.10 \mathrm{~T}=22,2^{\circ} \mathrm{C}$} \\
\hline distillate water & 0 & 0 & 0.50 & 0.30 & 0.10 & 0 \\
\hline Nestle & 0.05 & 0.45 & 0.40 & 0.10 & 0 & 0 \\
\hline \multicolumn{7}{|l|}{$25.10 \mathrm{~T}=22,2^{\circ} \mathrm{C}$} \\
\hline distillate water & 0 & 0.05 & 0.10 & 0.45 & 0.20 & 0.20 \\
\hline $\begin{array}{l}\text { Nestle } \\
\text { (opened on 23.10) }\end{array}$ & 0.05 & 0.2 & 0.55 & 0.15 & 0.05 & 0 \\
\hline
\end{tabular}

Table 4. Long-term changes in the activity of the "Ecolum" test culture in Nestle water (relative to distilled water) with preliminary stabilization of water under different conditions.*

Distilled Nestle Distilled Nestle Distilled Nestle Distilled Nestle Distilled Nestle water* $^{*}$ water water water water water water water water water

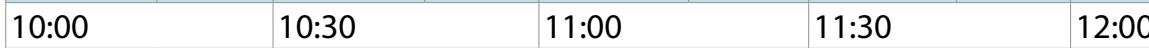

September 29 (kept in a laboratory room with catalytically active water)

\begin{tabular}{|l|l|l|l|l|l|l|l|l|l|}
\hline 170.6 & $\mathbf{7 . 3 3}$ & 294.6 & $\mathbf{3 . 5 2}$ & 286.2 & $\mathbf{3 . 0 8}$ & 210.4 & $\mathbf{3 . 0 5}$ & 223 & $\mathbf{2 . 9}$ \\
\hline 0.41 & 0.13 & 0.12 & 0.15 & 0.25 & 0.22 & 0.38 & 0.15 & 0.26 & 0.24 \\
\hline
\end{tabular}

October 02 - a laboratory experiment with no influence of external activating processes

\begin{tabular}{|l|l|l|l|l|l|l|l|l|l|}
\hline 40.4 & $\mathbf{1 . 0 2}$ & 39.8 & $\mathbf{1 . 1 2}$ & 45.6 & $\mathbf{1 . 0 1}$ & 52.6 & $\mathbf{0 . 8 6}$ & 42.6 & $\mathbf{0 . 9 5}$ \\
\hline 0.5 & 0.15 & 0.21 & 0.15 & 0.21 & 0.11 & 0.16 & 0.08 & 0.21 & 0.12 \\
\hline
\end{tabular}

October 16 (kept in a laboratory room with catalytically active water)

\begin{tabular}{|l|l|l|l|l|l|l|l|l|l|}
\hline 38.4 & $\mathbf{4 . 9 8}$ & 86.2 & $\mathbf{2 . 1 2}$ & 109.2 & $\mathbf{1 . 4 6}$ & 110.6 & $\mathbf{1 . 3 3}$ & 92.8 & $\mathbf{1 . 4 5}$ \\
\hline 0.22 & 0.18 & 0.19 & 0.32 & 0.24 & 0.31 & 0.28 & 0.3 & 0.31 & 0.1 \\
\hline
\end{tabular}

October 18 (kept in a laboratory room with catalytically active water)

\begin{tabular}{|l|l|l|l|l|l|l|l|l|l|}
\hline 34.2 & $\mathbf{5 . 5 5}$ & 53.4 & $\mathbf{3 . 1 4}$ & 86.8 & $\mathbf{1 . 7 6}$ & 131 & $\mathbf{0 . 9 5}$ & 112.6 & $\mathbf{1 . 1 8}$ \\
\hline 0.11 & 0.13 & 0.31 & 0.19 & 0.09 & 0.19 & 0.53 & 0.15 & 0.26 & 0.21 \\
\hline
\end{tabular}

October 20 - with no influence of external activating processes

\begin{tabular}{|l|l|l|l|l|l|l|l|l|l|}
\hline 81.4 & $\mathbf{3 . 1}$ & 138.6 & $\mathbf{1 . 9 2}$ & 126.6 & $\mathbf{2 . 2 2}$ & 276.2 & $\mathbf{0 . 8 5}$ & 277.8 & $\mathbf{0 . 8 5}$ \\
\hline 0.39 & 0.35 & 0.47 & 0.18 & 0.25 & 0.37 & 0.53 & 0.32 & 0.45 & 0.1 \\
\hline
\end{tabular}

October 23 - with no influence of external activating processes

\begin{tabular}{|l|l|l|l|l|l|l|l|l|l|}
\hline 226 & $\mathbf{1 . 8 2}$ & 339.6 & $\mathbf{1 . 3 2}$ & 288.4 & $\mathbf{1 . 2 9}$ & 211 & $\mathbf{1 . 2 6}$ & 306.6 & $\mathbf{1 . 0 8}$ \\
\hline 0.4 & 0.39 & 0.36 & 0.08 & 0.41 & 0.26 & 0.35 & 0.22 & 0.15 & 0.21 \\
\hline
\end{tabular}

Note * - The relative values of the luminescence intensity lo/lk (indicator of relative variation in the denominator)

** - Absolute values of the luminescence intensity of the culture in distilled water I, ppm (the culture is prepared on the day of research)

and the presence of activating systems. The range of changes in the activity of the test culture in distilled water varied from 43 to 307 ppm, which is associated with changes in the electromagnetic background and solar insolation in the noted period. Samples of Nestle water kept under the same conditions as distilled water, were characterized by higher values of Ecolum activity in the initial period of the experiment, but after 1.5 hours were similar 
with the activity of distilled water.

The exposure of Nestle water with no electromagnetic emitters present is characterized by approximately twice greater activity of the test culture, which can be a confirmation of the greater stability of metastable water states in absence of radiation.

The obtained background values of changes in the structural-physical and electrochemical parameters of water and the activity of the test microorganism culture can serve as independent criteria for subsequent evaluation of biological activity of water under the influence of electromagnetic radiation.

\section{Emission of low-frequency ER from Nestle water after its activation by UHF-radiation}

As noted above, water as a superconductor has the ability to emit electromagnetic radiation in a wide range of wavelengths and demonstrates self-organized critical behavior, which ensures its hypersensitivity to weak variable signals.

The excitation of the electronic subsystem of water is possible at different frequencies coinciding with the generation bands both in the low and high frequencies (UHF), which allows observing the radio emission of water using simple physical methods.

Nestle water in original packaging (0.5 liter polymeric container) was installed on a radiometric sensor EFA-300, located on a dielectric stand, and exposed to UHF radiation. The emitter of UHF radiation (several grammes of mass) was attached to the side surface of the container. The generator was turned on manually, while its shutdown was automatic. To minimize interference, the operator left the room for the duration of exposure, with no switching of network sources in adjacent rooms.

Electromagnetic emission of water after its exposure to UHF-radiation is characterized by quasiperiodically excited generation outbursts observed with the use of a peak detector (FIGURE 3), at the following frequencies: 0-1; $8 ; 12 ; 16 ; 20,3 ; 24,6 ; 39,8 ; 74 \mathrm{Hzv}[4]$.

With a longer observation of low-frequency emission of UHF-irradiated water (FIGURE 4), both delayed and retarded and advanced time of decreasing water activity and its subsequent

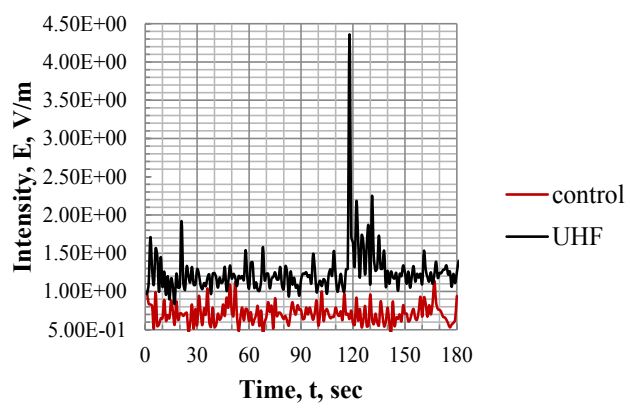

FIGURE 3. A fragment of recorded lowfrequency water emission (using a peak voltage detector in the frequency range of 5 $\mathrm{Hz}-2 \mathrm{kHz}$ ) after a 5-minute irradiation of the sample with UHF-radiation and a background water emission recorded $\mathbf{1 0}$ minutes prior to exposure.

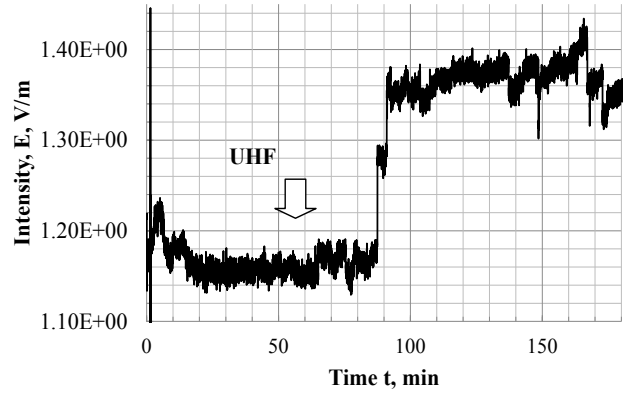

FIGURE 4. A low-frequency emission from water (rms detector, $5 \mathrm{~Hz}-2 \mathrm{kHz}$ range) in the repeated experiment with a longer recording of the signal (duration of UHF-radiation - 5 minutes).

sharp outburst, which lasts for a long time. No significant changes in the electromagnetic emission are observed during a 5 minute UHFirradiation, except for a general decrease in the emission intensity. In addition, activity drops are recorded in the LF emission of irradiated water, apparently associated with the periodic reorganization of the system of associates.

Considering the nature of the emission outbursts, it can be noted that most frequent are peaks with a relatively small amplitude, while high-amplitude peaks appear with large time intervals. According to [4], the characteristic features of appearance of electromagnetic emission of metastable states of water associates are the effects of a fairly rapid restructuring of their electronic activity present in a prolonged electromagnetic afterglow. In context of biological processes associated with structural changes in the phase of associated water, even more interesting is the temporal dynamics of electromagnetic emission, which is characterized 
by recurring events generated by changes in the electronic activity of the superconducting state. The recurrence of events in time (as well as in space) is a consequence of quantum nonlocality manifested in the self-reproducing property of the system (in this case, the water in the associated phase portion). In fact, this effect reflects the properties of quantum states of the quasiparticles (in physics this phenomenon is called "fractional revivals" [12]) acquired in the process of physical activation, which ensures their translation into the environment and long reproducibility in time.

Therefore, water associates (their electronic component) as macroscopic quantum objects contain in themselves the entire volume of electronic information about the object, which they transfer to the quantum-conjugate center. This property of quantum systems is of fundamental importance for biological processes due to the fact that electronic information transferred to the body in the form of an electronic crystal serves as a kind of "guidepost" that determines the direction of biochemical reactions.

\section{- Dynamics of electrochemical, structural-physical and biological characteristics of "Nestle" water after being activated by UHF emission of low intensity}

The presence of nonlocal interaction effects in advanced and delayed changes of the water emission characteristics during treatment by UHF emission stipulates the need for measuring of the changes in the structural-physical water state over longer periods. This will allowto correlate the structural-physical changes in the water with its LF emission characteristics and bioactivity.

After UHF emission treatment, during the first day of exposure some the redox potential, which has also appeared in the day before the water treatment (TABLE 6), values showed some increase. After 6 hours, Eh values of water began to drop pointing to self-induction of reactive oxygen intermediates. Maximum activity of the water is logged after twenty-four hours of exposure; it appears as synchronous change of electrochemical values and concentration of the peroxide anion radicals with deoxidizing activity in the water.
In the change dynamics of electrochemical characteristics of the analyzed "Nestle" water, periods qualitatively matching the concentration dependence of the peroxide anion-radical induction are noted (TABLE 2, FIGURE 2 and 3).

The analysis of variation changes in electrochemical characteristics presented in TABLE 5 confirms their correlation with the appearance of the anion-radical oxygen forms in the system. The latter are formed during the electron quantum condensation processes registered as the electromagnetic emission peaks. Here, the variation ratio of the peroxide anionradical concentration becomes high. Only after the excited system is left to stand for a prolonged period of time, the electron condensation rate slows down and the variation value normalizes (not exceeding 10\%).

It should be noted in the analysis of the dynamic changes in the structural-physical state of the water (with the results presented in TABLES 6 and 7) that the water structural organization shows only slight changes on the day of treatment and the preceding day before treatment. An exception is that on the day of the water treatment by UHF radiation, the highenergy states $\left(\mathrm{q}_{\mathrm{i}}=0.8-1.0 \%\right)$ presented by the peroxide anion radicals disappear in the energy distribution of the associated water phase.

On the day following the water treatment, the associated water phase content increases up to $\mathrm{q}_{\mathrm{av}}=0.587 \%$ (as compared with $\mathrm{q}_{\mathrm{av}}=$ $0.443 \%$ the day before). The weight ratio of the associated water phase increases up to $20 \%$ due to newly appeared high-energy states with $\mathrm{q}_{\mathrm{i}}>0.8 \%$ by means of conversion of the hydroxyl anion- into peroxide radicals.

Thus, the electronic dynamics of SP-state of the associated water phase subjected to UHF excitation, which shows response in LF electromagnetic emission, is accompanied by the structural-physical and electrochemical changes in the water medium.

FIGURE 5 shows the dynamic changes in the microorganism radiation rate after water treatment by the UHF emission. The peak value distribution of the microorganism luminescence intensity is characterized by a high variability. Bacteria luminescent emission intensity can be 
changed by an order of magnitude or more as compared with the background values during the whole exposure period. This indicates that the changes in the microorganism luminescence intensity is influenced by the periodic generative activity of the electromagnetic emission and associated processes of the electric charge transfer (electrons). Such changes of bioactivity variability are also observed in water when evaluating peroxide anion radicals content (Table 5), the fact of which indicates correlation of these processes.

Water treatment by the UHF emission leads to decrease of microorganism activity within several hours after the exposure (FIGURE 5); this is also represented by the nonlocal influence on the control water with the same composition as the water exposed to the emission ["Nestle" water in both cases] (FIGURE 6). As it follows from the graph, the microorganism activity decreases both in the control water and in the test water. This indicates that the control water responds to the structural-physical changes appeared in the test water after exposure to the UHF emission.

On the second day after water treatment by UHF emission, an anomalous increase of microorganism activity was observed in all analyzed water samples which exceeded the background values by an order of magnitude.

Table 5. Electrochemical characteristics and concentration of the peroxide anion-radicals in "Nestle" water after its activation by the UHF emission.

\begin{tabular}{|c|c|c|c|c|c|c|c|}
\hline \multirow{3}{*}{$\begin{array}{c}\text { Measurement } \\
\text { intervals, days }\end{array}$} & \multirow{3}{*}{$\begin{array}{c}\text { Time of } \\
\text { measurement, } \\
\text { hour:min }\end{array}$} & \multicolumn{4}{|c|}{ Electrochemical characteristics } & \multirow{2}{*}{\multicolumn{2}{|c|}{$\begin{array}{c}\mathrm{HO}_{2}^{-(*)} \\
\text { concentration, } \\
\mu \mathrm{g} / \mathrm{l}\end{array}$}} \\
\hline & & \multicolumn{2}{|c|}{ pH value } & \multicolumn{2}{|c|}{$\begin{array}{c}\text { Redox potential, } \\
\text { Eh, } \mathbf{m V}\end{array}$} & & \\
\hline & & average & var. & average & var. & average & var. \\
\hline \multirow{4}{*}{0} & 11:15 & 7.62 & 0.019 & 231.9 & 0.08 & $<0.1$ & \\
\hline & 12:45 & 7.39 & 0.004 & 233.2 & 0.015 & $<0.1$ & \\
\hline & 14:00 & 7.36 & 0.001 & 244.9 & 0.014 & $<0.1$ & \\
\hline & $15: 45$ & 7.33 & 0.004 & 248.7 & 0.011 & 0 & \\
\hline \multirow{4}{*}{$\begin{array}{l}1 \\
\text { UHF emission } \\
\text { treatment } 8: 20- \\
8: 25\end{array}$} & 11:00 & 7.09 & 0.001 & 259.7 & 0.006 & 0 & \\
\hline & $12: 50$ & 7.11 & 0.002 & 256.4 & 0.008 & 0 & \\
\hline & $14: 15$ & 7.29 & 0.005 & 234.1 & 0.015 & 5.39 & 0.55 \\
\hline & $15: 20$ & 7.25 & 0.001 & 235.5 & 0.010 & 1.59 & 0.39 \\
\hline \multirow{6}{*}{2} & 11:50 & 7.40 & 0.007 & 200.3 & 0.013 & 1.47 & 0.65 \\
\hline & 12:50 & 7.48 & 0.003 & 198.3 & 0.022 & 0.95 & 0.9 \\
\hline & 13:50 & 7.52 & 0.002 & 191.0 & 0.015 & 9.19 & 0.41 \\
\hline & 15:15 & 7.53 & 0.003 & 190.4 & 0.017 & 7.43 & 0.35 \\
\hline & 16:00 & 7.57 & 0.001 & 189.6 & 0.016 & 4.95 & 0.22 \\
\hline & $16: 55$ & 7.58 & 0.001 & 189.1 & 0.020 & 3.19 & 0.41 \\
\hline 3 & 11:55 & 7.25 & 0.002 & 197.8 & 0.012 & 2.16 & 0.11 \\
\hline 3 & 11:55 & 7.25 & 0.002 & 197.8 & 0.012 & 2.16 & 0.11 \\
\hline
\end{tabular}

Table 6. Changes in the associated phase $\left(q_{z v}, \%\right)$ of Nestle water after treatment by the UHF emission.

\begin{tabular}{|c|c|c|c|}
\hline \multirow{2}{*}{ Measurement intervals, days } & \multirow{2}{*}{ Impact } & \multicolumn{2}{c|}{\begin{tabular}{c} 
Phase part, qav.,\% \\
\cline { 3 - 4 }
\end{tabular}} \\
\cline { 3 - 4 } average & variations \\
\hline 0 & Without impact (control) & 0.442 & 0.038 \\
\hline 2 & UHF treatment 8:20-8:25 & 0.443 & 0.032 \\
\hline 3 & after 24 hours & 0.587 & 0.028 \\
\hline & after 2 days & 0.468 & 0.025 \\
\hline
\end{tabular}

Note $^{*}$ - the temperature is $20,6 \pm 0,2^{\circ} \mathrm{C}$ (opened water samples were placed in climatostat) 
Thus, the metastable state of "Nestle" water influences the structural-physical state of the water in the immediate environment by way of nonlocal interaction processes and water aerosol constituent excitation [4].

Long-term monitoring of the dynamic changes in the water bioactivity (FIGURE 7) shows that the maximum water bioactivity appears in the second day after treatment by UHF emission. At the same time, water medium excitation represented by bioactivity increase appears before impact of the UHF emission (see

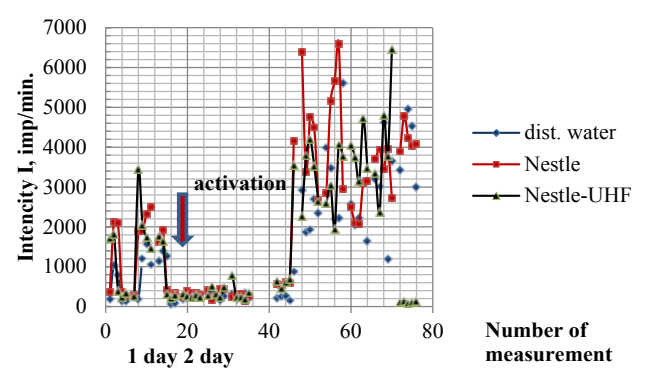

FIGURE 5. Response of "Ecolum" microorganisms to a 5-minute UHF emission depending on the exposure ( $I$ is emission intensity without averaging). series 2, day 0). After emission exposure, the gradual decrease of water bioactivity is registered and the maximum influence of emission appears only on the second day.

This progression of the process is stipulated by the changes in structural-physical characteristics of the associated water phase connected to generation of reactive oxygen intermediates resulting in change of electrochemical water characteristics.

When analyzing the obtained results, we can conclude that the biological effects demonstrated by the activated water are stipulated by the charge transfer to the primary electron receptors in the organism initiating structural and dynamic processes of hydration and conformational reconstruction of biopolymers (phospholipids) [22,23]. Different trends of "Ecolum" microorganism reaction in the after-treatment by UHF emission stages are associated with the processes of growth or degradation of the associated water phase andredistribution of the volume charge $[24,25]$

\begin{tabular}{|c|c|c|c|c|c|c|c|c|}
\hline \multirow{2}{*}{$\begin{array}{l}\text { Measurement } \\
\text { intervals, days }\end{array}$} & \multicolumn{8}{|c|}{ Energy levels, $\Delta$ qi, $\%$} \\
\hline & $0 \ldots 0.2$ & $0.2 \ldots 0.4$ & $0.4 \ldots 0.6$ & $0.6 \ldots 0.8$ & $0.8 \ldots 1.0$ & $1.0 \ldots 1.2$ & $>1.0$ & $>1.2$ \\
\hline 0 & 0.05 & 0.35 & 0.25 & 0.25 & 0.1 & 0 & 0 & 0 \\
\hline $\begin{array}{l}1 \text { UHF treatment } \\
8: 20-8: 25\end{array}$ & 0.05 & 0.3 & 0.45 & 0.2 & 0 & 0 & 0 & 0 \\
\hline 2 (after 24 hours) & 0 & 0.1 & 0.55 & 0.15 & 0.15 & 0.05 & 0.05 & 0 \\
\hline 3 (after 2 days) & 0.05 & 0.3 & 0.4 & 0.25 & 0 & 0 & 0 & 0 \\
\hline
\end{tabular}

Note * - the experimental conditions are the same as in Table 7.

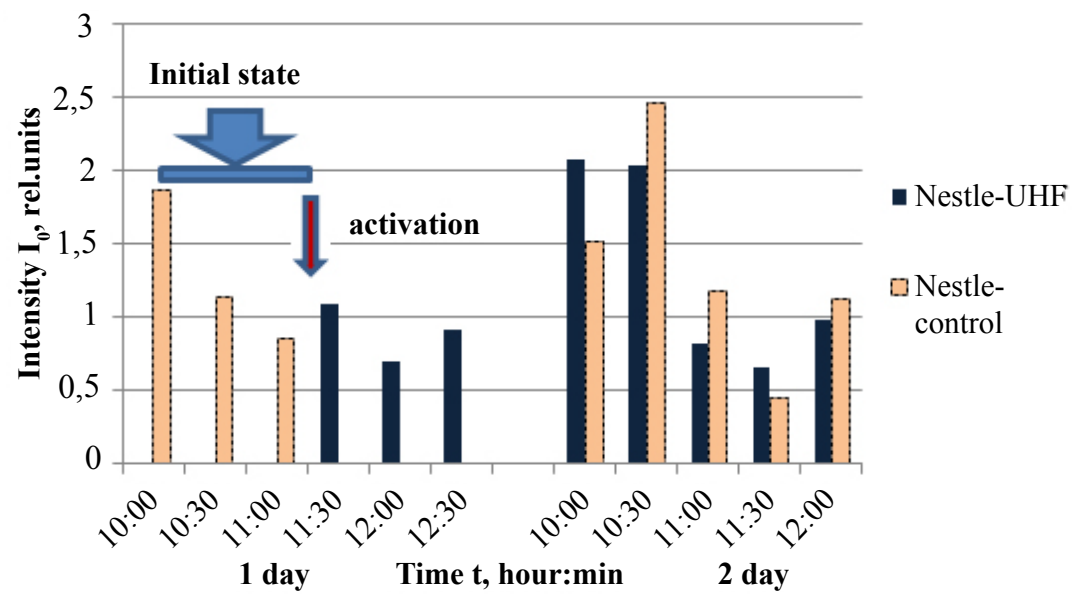

FIGURE 6. Dynamic changes in E.Coli luminescent emission intensity in Nestle water treated by UHF emission, relatively distilled water (Io=In/Id where In, Id - intensity of Nestle water samples and distilled water, correspondingly, exposure time is $\mathbf{5}$ minutes). 


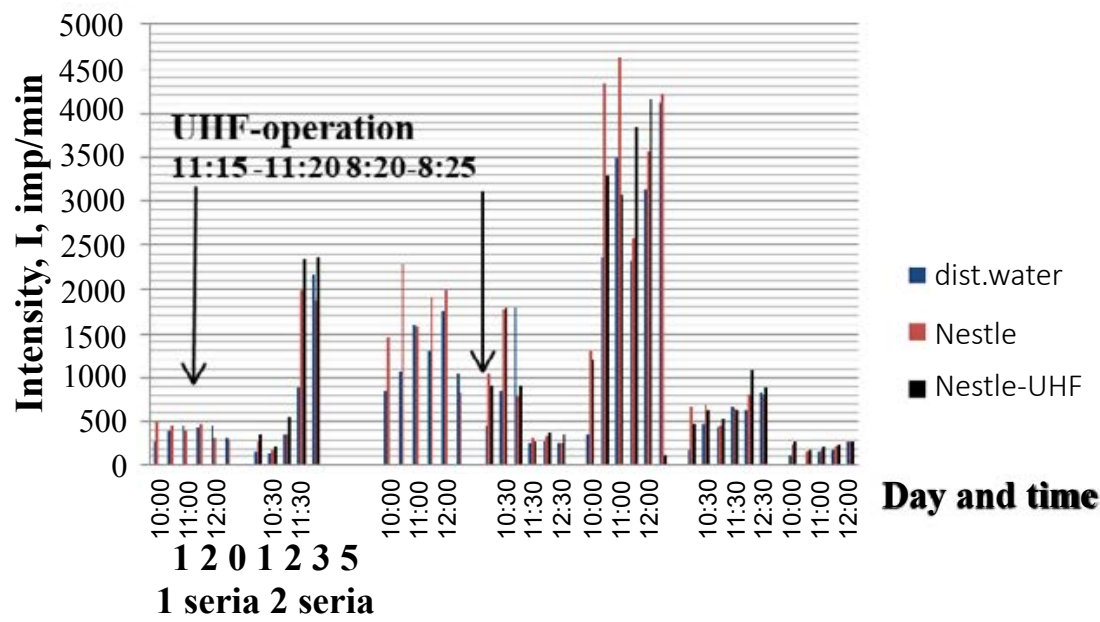

FIGURE 7. Dynamic changes of luminescent I emission intensity of "Ecolum" microorganisms exposed in water treated by the UHF emission (two series of experiments spread out over 1 week).

oscillating in macroscopic timescales determined by the quantum nonlocality effects.

But such significant biological effect of the microorganism activity increase (by more than decimal exponent) in the medium without metabolites cannot be associated solely with the structural changes of hydrate water under the influence of UHF emission.

At the same time, according to the works [26-28], increase in luminosity of the cell bacterial luciferase is stipulated by production of ATP, the universal biological energy carrier. In prokaryotic cells, ATP is produced by protonic ATP synthase (F0F1-ATP-synthase where FO-, F1 are protein complexes) built into the cell membrane. According [29], the key elements in the activity of F0F1-ATPsynthase proton channels are the negatively charged amino-acid residues of $\diamond$ and $>$ subunits containing protonated groups able to retain protons and transfer them between each other (proton motor) [29-33]. But these works do not describe the mechanism making $\gamma$-subunits to rotate together with $\varepsilon$ and and $\delta$-subunits of F0 complex in the absence of a proton gradient on membrane. Based on our results concerning the influence of structural and physical state of the associated water phase on the activity of
F0F1-ATP-synthase of microorganisms, it is assumed that ATP-synthase operating efficiency is determined by the electronic activity of water with behavior described in scientific terms of the high temperature SP-state [4].

On this basis, the rolling movement of F0 complex oligomer subunits is based on the electron entry on the hydrate structures of $\alpha$-helical proteins of the cell membrane forming the rotary magnetic field (a characteristic of SPstate) which interacts with the magnetic domain of $\gamma$-subunit of $\mathbf{F O}$ complex and makes it rotate. This results in periodic excitation of hydrationdehydration processes resulting in formation of protons in the system which enter the active centers of $\mathbf{F} \mathbf{1}$ complex. Besides, these processes create conditions for quantum condensation of electrons from the environment which support rotation of the cell motor.

Thus, the cell motor functioning depends on the degree of water hydration in the cell supported by the metabolic electron processes with the environment. Therefore, the associated water phase being a quantum oscillator operates as an energy supplier for the cell, while also responsible for transforming thermal energy into chemical and mechanical energy. 


\section{REFERENCES}

Kuznetsov DB. Development of physicochemical methods of improvement of the efficiency of probiotic bacterial cultures cultivating. A dissertation. (2014).

Torgomyan H. Low intensity electromagnetic irradiation with 70.6 and $73 \mathrm{GHz}$ frequencies affects Escherichia coli growth and changes water properties. Cell Biochem. Biophys.60(3), 275-281 (2011).

Kargatova TV. Evaluation of survival of transgenic strain Escherichia coli Z905/pPHL7 in water microcosms. A dissertation. Krasnoyarsk. (2005).

Stehin AA, Yakovleva GV, Pronko $\mathrm{KN}$, Zemskov VM. The water as a main component of biochemical processes. Clin. Pract.15(5), (2018)

Stehin AA, Yakovleva GV. Structured water: Non-linear effects. M. LKI, (2008) Russia.

Samuilov FD, Nikiforov EA, Nikiforova VI. Nuclear magnetic relaxation water of protons in plant cells in different growth phases. DAN. 357(5), 700-703 (1997).

Zenin SV. Structural study of water by proton magnetic resonance method. Reports of RAS. 332(3), 328-329 (1993).

Daniloc OB, Belousova IM, Mak AA, et al. Studies of the singlet oxygen generation using optically pumped fullerenes. Opt. Spectrosc.

Allen CB, Schneider BK, White CJ. Limitations to oxygen diffusion in invitro cell exposure systems in hyperoxia and hypoxia. Am. J. Physiol. Lung. Cell. Mol. Physiol. 281(4), L1021-L1027 (2001).

Bunkin AF, Nurmatov AA, Pershin SM. Coherent four-photon spectroscopy of low-frequency librations of molecules in a liquid. UFN.176(8), 883-889 (2006).

Semihina LP. Low-frequency dielkometry of liquids in weak electric curl fields. A dissertation. Moscow. (2006).

RahmaninYuA, Stehin AA, Yakovleva GV. Water biophysics: quantum non- locality in water treatment technologies; regulatory role of the associated water in the cellular metabolism; rationing of bioenergetics activity of the drinking water. Moscow. LENAND. (2016).

Petrosyan VI. Radiophysics of water and life. Water, paradoxes and grandeur of small values. Saarbrücken. Lambert Academic Publishing. (2017).

RahmaninYuA, Kondratov VK, Mihaylova RI, Stekhin AA, Yakovleva GV. The water is a cosmic event: cooperative properties and biological activity (second revised and enlarged edition). Karlovy Vary, CZ, Tinowa printing office. (2014).

Kuznetsov AN. Semi-conductor injection oscillator based on A3B5 heterostructure. Patent of RF No. 134365 dated 16.04.2012.

Danilov VS. Bioluminescent sensing systems on the basis of lux operons of different luminescent bacteria. MSU Bulletin. Ser. 3, 20-24 (2002).

Daniel FS. Development of luminescent sensing systems with clinical applications. A dissertation. Kentucky (2011).

MR 01.021-07 Methods of express determination of the integral chemical toxicity of drinking, surface, ground, sewage and treated sewage water using Ekolyum bacterial test. Russia.

Debouzy JC. Effetsbiologiques des rayonnementsmillimétriques $(94 \mathrm{GHz})$. Quellesconséquences à long terme? Pathologie. biologie. 55(5), 246-255 (2007).

Kolesnikov VG, Dreval NV. Application of combined impact of electromagnetic and acoustic waves on microalgae, in order to detect resonance frequencies. Phisikazhivogo. 18(3), 24-28 (2010).

Vasilev NS. Study of the low-frequency electromagnetic field impact on cultures Escherichia coli and Saccharomyces cerevisiae. A dissertation. Moscow. (2006) .

KrinitskayaAYu, Suhanov PP, SedelnikovYuE. Impact of extremely high frequency radiation on the structuraldynamic state of model biomembranes. Radio electronics. 4, 1-9 (2011).

Ovchinnikova K, Pollack GH. Can water store charge? Langmuir. 25(1), 542547 (2008).

Klimov A, Pollack GH. Visualization of charge-carrier propagation in water. Langmuir. 23(23),11890-11895 (2007).

Elia V, Napoli E, Niccoli M. Physicalchemical study of water in contact with a hydrophilic polymer. J. Therm. Anal. Calorim. 112(2), 937-944 (2013).

Sobolev AD, Ismailov AD, Danilov VS. Bioluminescence kinetics in bacterial luciferase reaction with various aliphatic aldehydes. Biochemistry. 54(2), 20612065 (1989.

Ismailov AD, Beria LV, Danilov VS. Formation of bacterial luciferase aldehydic substrate during lipid peroxidation induced by the ultraviolet light. Biochemistry. 59(4), 519-524 (1994).

Ismailov $\quad \mathrm{AD}$ Bacterial bioluminescence: Mechanisms of formation of aldehydic and flavin substrates and their interaction with luciferase. Dissertation. Moscow. (1995).

Romanovsky Yu M, Tikhonov AN. Molecular living cell energy converters. Protonic ATP synthase-rotating molecular motor. UFN.180, 931-956 (2010).

Figueroa XA, Pollack GH. Exclusionzone formation from discontinuous nafion surfaces. Int. J. Des. Nat. Ecodyn. 6(4), 286-296 (2011).

Pollack GH, Figueroa X, Zhao Q. The minimal cell and life's origin: Role of water and aqueous interfaces. The Minimal Cell.105-121 (2011).

Das R. Pollack GH. Charge-based forces at the nafion-water interface. Langmuir. 29(8),2651-2658 (2013).

Campbell ZT, Weichsel A, Montfort WR, Baldwin TO. Crystal structure of the bacterial luciferase: Flavin complex provides insight into the function of the subunit. Biochem. 48(26), 6085-6094 (2009). 